\title{
Stratégies familiales et qualité de vie au Mali à travers les données du recensement
}

\author{
Abdoul Moumouni Nouhou', Siaka Cissé ${ }^{2}$, Daouda Aba Fané ${ }^{3}$, Assa Gakou Doumbia ${ }^{4}$ et \\ Claudine Sauvain-Dugerdil ${ }^{5}$
}

\author{
${ }^{1}$ Institut de démographie et de socio-économie, Université de Genève \\ ${ }^{2}$ Institut National de la Statistique, République du Mali \\ Claudine.Sauvain@unige.ch
}

\section{Résumé}

Par une analyse du dernier recensement malien (2009), cette étude examine dans quelle mesure la configuration du ménage influence sa qualité de vie. Le postulat est que, selon leur composition, les ménages développent des stratégies familiales qui leur permettent d'accéder et de valoriser plus ou moins les ressources disponibles. Une typologie des ménages distingue cinq configurations urbaines et cinq rurales dont on teste les différences de qualité de vie, telle que mesurée par le degré de modernisation matérielle (indice de confort du logement) et la scolarisation ajustée des enfants. Les grands ménages élargis apparaissent nettement avantageux. Mais en milieu rural, ce sont les ménages dirigés par un chef instruit qui viennent en tête. Les ménages urbains dirigés par les femmes ne sont pas systématiquement vulnérables, se situant juste après les plus grands ménages. Dans les deux milieux ce sont les très petits, dirigés par des chefs plus jeunes ou plus âgés qui sont les moins bien lotis.

Mots clé : Qualité de vie, confort de logement, scolarisation ajustée, type de ménage, accès aux ressources

\section{Abstract}

\section{Family strategies and quality of life in Mali as described by the census}

This paper uses the data of the latest Mali census (2009) to examine whether the composition of the household influences its quality of life. We postulate that depending on their configuration, households develop varying family strategies to access and valorise the resources available. A typology identifies five urban and five rural household configurations. We analyse their differences in quality of life, the latter being measured by the degree of material modernization (comfort of housing) and the average schooling of children. Large extended households appear better off. However, in the rural settings, those headed by an educated head come first. The urban households headed by a woman do not appear to be especially vulnerable, as they rate just after the larger ones. In both settings, the worse situation is found among very small households with younger or older heads.

Keywords : Quality of life, comfort of housing, average schooling, type of household, access to resources.

\section{Introduction}

La relation entre la configuration des ménages et la qualité de vie a été principalement examinée à travers un lien supposé entre, d'une part, la pauvreté et une fécondité élevée et, d'autre part, une nucléarisation des familles comme conséquence de la modernisation des sociétés. Toutefois, en Afrique subsaharienne la grande famille reste une réalité; la taille des ménages s'est même accrue dans certains contextes défavorisés (Lee, 1999) et, actuellement au Mali, les ménages urbains sont légèrement plus grands que les ménages ruraux. La paupérisation, à la fois, accroît le rôle de refuge de la grande famille et la fragilise (Pilon et Vignikin, 1996).
La diversification des stratégies de subsistance est au cœur de la capacité de "bricolage productif" dont font preuves les populations sahéliennes (Batterbury, 2007), mais elle est aussi porteuse d'inégalités socioéconomiques croissantes entre les ménages. La montée des priorités individuelles, sous l'effet notamment de la crise économique et d'une urbanisation rapide, fragilise la cohérence des grandes familles et leur capacité à mieux utiliser les ressources que les ménages restreints (Toulmin, 1992). Néanmoins, ce nouvel individualisme "à l'africaine", ne signifie pas l'isolement des individus et la 
disparition des solidarités familiales (Marie éd. 1997, Calvès et Marcoux, 2007).

Nous examinons ici les avantages et désavantages des grands ménages, à savoir leur capacité à développer des stratégies qui se reflètent dans leur qualité de vie. Le ménage représente ainsi une unité socioéconomique qui ne correspond certes pas au réseau familial, mais à ce que nous avions appelé le "groupe familial visible", à savoir les proches avec lesquels on partage son quotidien (Sauvain-Dugerdil et al, 1997). Selon la terminologie de l'approche par les Capabilités (Sen, 1999), les caractéristiques des ménages sont considérées comme des "facteurs collectifs de conversion" qui permettent à cette unité familiale d'utiliser plus ou moins bien les ressources existantes pour réaliser la vie qu'elle valorise.

L'objectif du présent travail est donc de s'interroger sur les raisons qui font que, dans le contexte malien de grandes disparités résultant de mutations sociales profondes, certains ménages, selon leur configuration, profitent plus ou moins bien des (nouvelles) opportunités liées notamment à l'urbanisation et à la globalisation, et/ou surmontent plus ou moins bien les chocs endogènes et exogènes. L'hypothèse est que les ménages nucléaires auraient adopté des valeurs modernes et qu'ils seraient plus égalitaires, à savoir les rôles individuels seraient moins différenciés. En revanche, les plus grands ménages auraient de meilleures opportunités d'accès et d'utilisation des ressources. Grâce à leur maind'œuvre plus nombreuse, ils auraient une plus grande capacité à diversifier leurs stratégies de subsistance. Cependant, en milieu urbain, les grands ménages n'ont pas forcément un avantage car, dans un contexte d'individualisation, les solidarités faiblissent, les charges sont moins partagées et l'autorité du chef de ménage est mise en cause. Les opportunités seraient également modulées par les caractéristiques du chef de ménage, en particulier son niveau d'instruction et son sexe, ainsi que les caractéristiques de leurs membres, notamment le nombre de personnes dépendantes et la présence de migrants.

Revue de la littérature et cadre théorique

Des liens entre fécondité et pauvreté aux stratégies familiales d'accès aux ressources Une série de travaux a mis en évidence une stagnation dans la baisse de la fécondité en Afrique dès le milieu des années 1990 (Locoh 2002 ; Bongaarts 2006 ; Garenne 2013). Plus largement, de nombreuses études ont cherché à analyser la relation entre fécondité et pauvreté sans arriver à des conclusions simples. Comme le souligne Sandron (2013) dans sa revue de la littérature, la théorie de la modernisation reste une explication prégnante; les nombreux travaux, tant ceux relatifs à la transition démographique historique en Europe qu'aux évolutions actuelles n'ont toutefois pas fourni de preuves évidentes d'une corrélation entre les tendances de la fécondité et celles de la situation socioéconomique (Garenne, 2009), ni quels sont les facteurs qui modulent leurs inter-relations. L'analyse de Shapiro et al (2013) des tendances dans 49 pays ayant réalisé plusieurs enquêtes démographiques et de santé (EDS), dont 26 en Afrique subsaharienne, confirme l'association entre une diminution consistante de la fécondité, l'accroissement de la scolarisation des femmes et un déclin antérieur de la mortalité infanto-juvénile. En revanche, une croissance rapide du PIB apparaît au contraire liée à un déclin plus lent de la fécondité. Sur la base d'une compilation de 42 études et d'une analyse plus approfondie dans deux contextes, Schoumaker et Tabutin (1999) avaient déjà mis en évidence l'influence des définitions retenues et le rôle d'autres facteurs tant sur la fécondité, à travers par exemple l'âge à la première maternité, que sur la situation socio-économique. Ainsi, comme le relève Leridon (20|4), le constat de Dirk Van de Kaa selon lequel " il n'existe pas encore une histoire probante unique qui ferait consensus auprès de l'ensemble de spécialistes, sur le cadre et les conditions nécessaires et suffisantes pour générer une influence majeure sur l'évolution de la fécondité " (Van de Kaa, 1996, p.390) reste certainement valable. Pour reprendre les termes de Billari et Wilson (200I) au sujet de l'Europe occidentale, c'est plutôt à une "convergence vers la diversité" que l'on assiste.

Finalement, c'est aussi la mesure de la pauvreté qui est largement revisitée dans la ligne du concept de développement humain. Suivant les travaux de l'économiste Amartya Sen, les mesures monétaires de la pauvreté sont de plus en plus remplacées, ou du moins complétées, par des indicateurs multidimensionnels. Plus largement, sa perspective des Capabilités (Sen, 1999; Nussbaum et Sen, 1993; Robeyns, 2005), qui se concentre sur l'éventail des possibilités plus que le résultat, ne conçoit pas la qualité de vie comme une résultante simple du manque de ressources locales, mais de facteurs multiples qui font que les individus et les familles ont plus ou moins accès à ces ressources et plus ou moins la capacité de les exploiter à leurs fins, c'est-àdire, selon les termes de Sen de " vivre la vie que l'on a raison de valoriser ". La pauvreté est alors avant tout le résultat des inégalités d'accès aux ressources; les plus démunis ne peuvent valoriser les nouvelles opportunités et n'arrivent pas à surmonter les nouvelles contraintes. L'application de l'approche par les Capabilités aux études démographiques vise donc à mieux comprendre les disparités croissantes 
de développement humain et sélectionner les meilleurs indicateurs pour identifier les inégalités d'opportunités dans des sociétés en transformations rapides (Sauvain-Dugerdil, 20|4).

II convient donc de dépasser les conclusions de causalité simple. De nombreux études soulignent l'importance de prendre en compte les dynamiques distinctes de sous-populations selon le lieu de résidence (voir par exemple Garenne, 2013 et Shapiro et al, 20|3). De plus en plus nombreux sont aussi les travaux qui mettent en évidence le rôle de la croissance des inégalités sociales dans les périodes de transition (Sandron, 20/3). Mais, surtout, les constats d'évolutions globales concordantes ou discordantes ne permettent pas de mettre en évidence les facteurs à l'œuvre. C'est au niveau des familles que les stratégies de survie se définissent, et au niveau des individus que les comportements se concrétisent. La famille représente l'unité sociale de base et, donc, le niveau le mieux approprié pour l'analyse du lien entre la fécondité et la qualité de vie. Ainsi, au-delà du nombre d'enfants, c'est leur rôle dans les stratégies familiales, notamment les différentes facettes de la fonction assurantielle (LeGrand et al, 2003), qui sont à examiner.

Les présentes évolutions démographiques et socioculturelles modifient profondément les systèmes familiaux. La transformation des systèmes de subsistance et la diffusion de la scolarisation font évoluer le rôle de l'enfant, d'une main d'œuvre qui rapporte à un investissement qui coûte (Ariès, 1960; Caldwell, 1982). "L'ébranlement des modèles anciens" et les crises économiques contribuent à éroder les solidarités intergénérationnelles (Locoh et Mouvagha-Sow, 2005). Néanmoins, une série de travaux montrent que les grandes familles sont une source de richesse et diminuent les risques de précarité (Toulmin, 1992). D'autre part, comme l'avait montré Maria-Eugenia Cosio-Zavala (200I) pour les villes latino-américaines, la petite famille peut être le résultat de progrès mais aussi de contraintes : pour les secteurs privilégiés de la population, la baisse de la fécondité exprime une amélioration du statut de la femme, alors que pour d'autres, elle représente un malthusianisme de la pauvreté, à savoir les conséquences de la précarité urbaine.

\section{Données et méthodes :}

le ménage comme unité d'étude

Nous examinons le cas du Mali, un pays dans lequel la fécondité n'a connu qu'une diminution minime, passant de 7,I enfants par femme dans les années 1980, à 6,8 en 2010-I 2 (World Population Prospect). En milieu urbain, elle stagne à 5,5 depuis les années 1990. En moyenne, la taille des ménages - 6,2 en
2009 - s'est accrue au cours des trente dernières années $(5, I$ en 1976), mais à peine durant la dernière décennie (6, en 1998) $)^{i i}$; elle est légèrement supérieure en milieu urbain (6,3 contre $6, I$ personnes en milieu rural). Globalement, les ménages nucléaires sont majoritaires $(5 \mid \%)$, suivis de ceux élargis aux personnes apparentées (32\%). Les chefs de ménage sont généralement des hommes, seuls $13 \%$ sont dirigés par une femme ( $16,8 \%$ en milieu urbain). Dans l'ensemble, la plupart des CM n'ont aucun niveau d'instruction $(76,7 \%)$ et seulement $4,8 \%$ d'entre eux ont atteint le niveau secondaire, le milieu de résidence jouant un grand rôle de différenciation à ce niveau.

Les liens entre les systèmes familiaux et la qualité de vie au Mali sont analysés ici avec les données du Recensement Général de la Population et de l'Habitat réalisé en 2009 (RGPH 2009). Par leur nature, les données de recensements comportent certaines faiblesses. Elles fournissent une photographie du moment pour un nombre limité de caractéristiques qui ne permet donc ni une analyse des changements, ni un examen approfondi des facteurs explicatifs. D'autre part, les données souffrent de certaines imprécisions. C'est ainsi que le rapport de l'INSTAT consacré à l'évaluation de la qualité des données iii souligne des problèmes d'attraction aux chiffres ronds, de mémoire des événements plus anciens, d'effet de télescopage notamment pour les naissances des 12 derniers mois, de transferts d'âge de certaines femmes au-delà des limites établies, des incohérences entre les naissances totales et celles des 12 derniers mois. Un recours à des techniques spécifiques d'ajustement a été nécessaire pour corriger ces incohérences et estimer la structure et le niveau de la fécondité.

Au-delà de ces imprécisions, les données du recensement ont le grand avantage de fournir une image détaillée de la répartition spatiale des ménages et des facteurs socio-économiques associés. Par leur exhaustivité, elles fournissent des effectifs suffisants pour mener des analyses même au sein de petits groupes socio-économiques qu'il n'est pas possible de distinguer avec les données d'enquêtes.

L'objectif est ici de se situer au niveau des ménages, c'est-à-dire de construire une typologie des ménages et des indicateurs de qualité de vie du ménage.

Caractérisation des ménages maliens avec les données du recensement

Le concept d'attributs du ménage, que nous proposons ici, renvoie à la prise en compte de la diversité des configurations des ménages, en s'interrogeant sur les caractéristiques qui expriment le mieux les disparités entre les ménages maliens. Audelà de la taille du ménage et des seules caractéristiques de leur chef, ce concept intègre les 
interactions entre les caractéristiques individuelles des différents membres en présence. Nous distinguons les attributs qui expriment les disparités d'opportunités résultant de la composition du ménage et, d'autre part, les inégalités d'accès aux ressources selon les caractéristiques et situations de leurs membres. Les modalités ont été choisies selon les distributions des variables, mais aussi dans le but de distinguer les situations relativement marginales (Tableau I).

Tableau I. Récapitulatif des variables d'attributs du ménage

\begin{tabular}{|c|c|c|c|}
\hline Attributs & Variables & Valeurs exclues & Modalités \\
\hline Opportunités offer & rtes par la composition & du ménage & \\
\hline $\mid \begin{array}{l}\text { Taille } \\
\text { composition }\end{array}$ & tTaille du ménage & $>25$ personnes & $\begin{array}{l}\text { Très petit }(I-2) \\
\text { Petit }(3-5) \\
\text { Grand }(6-8) \\
\text { Très grand }(9 \text { et plus) } \\
\end{array}$ \\
\hline du ménage & Type de ménage & & $\begin{array}{l}\text { Nucléaire } \\
\text { Elargi autres parents } \\
\text { Elargi autres parents et/ou sans liens }\end{array}$ \\
\hline & & Pour chacun des sexes & \\
\hline Structure & Enfants (6-I4) & $>7$ & \\
\hline & $\begin{array}{l}\text { Jeunes/Adultes (I5- } \\
44)\end{array}$ & $>10$ & 3 et plus \\
\hline Fécondité & Parité relative & $\begin{array}{l}\text { Nb nais. vivantes } \\
>6,8,10, \quad 11,12 \text { \& } 13 \\
\text { pour femmes âgées de : } \\
20-24,25-29,30-34, \\
35-39,40-44 \\
\& 45-49 \text { ans }\end{array}$ & $\left\{\begin{array}{l}\text { Nulle } \\
\text { Faible }(>0 \text { et }<=0,72)^{\text {iv }} \\
\text { Intermédiaire } \\
\text { Elevée }(>=1,55) \\
\text { Pas de femmes } 20-49 \text { ans }\end{array}\right.$ \\
\hline & Sexe du CM & - & Homme - Femme \\
\hline Caractéristique du & $\begin{array}{l}\text { Niveau d'instruction } \\
\text { du CM }\end{array}$ & - & \begin{tabular}{|l} 
Aucun \\
Primaire \\
Secondaire ou plus
\end{tabular} \\
\hline $\mid \begin{array}{l}\text { Cher de menage } \\
(\mathrm{CM})\end{array}$ & Age du CM & - & $\begin{array}{l}\text { Moins de } 25 \text { ans } \\
25-44 \text { ans } \\
45-59 \text { ans } \\
60 \text { ans ou plus }\end{array}$ \\
\hline Accès aux ressourc & ces selon les caractéristi & tiques des membres du m & lénage \\
\hline & $\begin{array}{l}\text { Existence d'enfants } \\
\text { travailleurs }\end{array}$ & & $\begin{array}{l}\text { Oui - Non } \\
\text { Pas d'enfants 6-14 ans }\end{array}$ \\
\hline $\begin{array}{l}\text { Statut } \\
\text { d'occupation }^{\vee}\end{array}$ & $\begin{array}{l}\text { Personnes de } 15 \text { à } 98 \\
\text { ans occupées }\end{array}$ & $\begin{array}{l}\text { Ménage avec } \\
>15 \text { adultes occupés }\end{array}$ & $\begin{array}{l}0 \\
\text { I à } 2 \\
3 \text { ou plus } \\
\text { Pas de membres de } 15 \text { ans et plus }\end{array}$ \\
\hline Migration ${ }^{\mathrm{vi}}$ & \begin{tabular}{|l} 
Emigrants \\
Migrants \\
internationaux \\
Migrants internes \\
\end{tabular} & $\begin{array}{l}\text { Emigrants et migrants } \\
\text { internationaux }>9 \\
\text { Migrants internes }>15\end{array}$ & Oui \\
\hline & & Pour chacun des sexes & Hommes \\
\hline $\begin{array}{l}\text { Groupes } \\
\text { d'âges non actifs }\end{array}$ & $\begin{array}{l}\text { Petits } E \text { et enfants ( } 0- \\
\text { I4) } \\
\text { Agés ( } 65 \text { et plus) }\end{array}$ & $\begin{array}{l}>5 \\
>3\end{array}$ & $\begin{array}{l}0 \\
\text { là2 } \\
3 \text { et plus }\end{array}$ \\
\hline Actifs matures & Seniors (45-64) & $>4$ & $\begin{array}{l}\text { Oui } \\
\text { Non }\end{array}$ \\
\hline
\end{tabular}


A travers une analyse factorielle des correspondances multiples (AFCM), nous avons identifié les attributs qui discriminent le plus les ménages vii. L'examen de la distribution des valeurs des contributions des différentes variables selon les trois premiers facteurs (qui expliquent $21 \%$ de la variabilité totale) permet de dégager les principales caractéristiques qui distinguent les ménages maliens. Ainsi, le premier axe, distingue essentiellement les ménages selon leur taille et les caractéristiques qui y sont directement associées (nombre de jeunes et nombre d'adultes). Le second facteur distingue, parmi les très petits ménages, un groupe atypique formé de ceux n'ayant aucun adulte, aucun actif occupé, souvent aucun petit enfant, mais des personnes âgées. Les caractéristiques qui contribuent le plus à la variance du troisième axe factoriel sont le niveau d'instruction du chef de ménage, la mobilité des membres du ménage, la présence de personnes non apparentées au chef de ménage et, surtout, le milieu de résidence. La description bivariée ${ }^{\text {vii }}$ montre cependant que les différences entre les ménages urbains et ruraux sont complexes. Bien que la fécondité soit supérieure en milieu rural, globalement la taille moyenne des ménages $y$ est légèrement inférieure et, contrairement à la thèse de la modernisation, c'est en milieu rural que les ménages nucléaires sont plus nombreux. C'est en milieu urbain que l'on trouve les ménages atypiques par leur taille - très petits ou très grands -, mais aussi ceux qui comptent plus d'adultes et plus d'adultes occupés, plus de migrants et des CM plus instruits. En milieu rural, résultats vraisemblables de l'émigration vers les villes, les ménages ont moins d'adultes et un peu plus de seniors et d'âgés et plus de chefs aux caractéristiques atypiques (femmes, jeunes et âgés). Le ménage compte plus d'enfants et ceux-ci sont plus souvent travailleurs qu'en milieu urbain. Ces distinctions confirment que, dans un pays comme le Mali, les sociétés rurales et urbaines ont des situations et des modes de vie distincts, raison pour laquelle il importe de les analyser séparément.

Une typologie des ménages a été construite sur la base des attributs relatifs à la composition du ménage, à savoir la taille et le type du ménage, sa structure par âge décrite par le nombre d'enfants et celui de jeunes et d'adultes, et les caractéristiques du chef de ménage (sexe, âge et niveau d'instruction). $\mathrm{Vu}$ les fortes corrélations entre les différentes caractéristiques, les calculs ne sont pas appliqués directement sur celles-ci, mais sur les facteurs issus de l'AFCM, lesquels, par définition, résument des informations indépendantes. L'ensemble des analyses est réalisé séparément pour le milieu rural et le milieu urbain en retenant les huit premiers facteurs qui correspondent au $3 / 4$ de la variabilité totale des ménages $(74 \%$ en milieu rural et $73 \%$ en milieu urbain). Les meilleurs modèles sont, dans les deux cas, ceux avec cinq clusters que nous classons ici par effectifs décroissants. Dans chacun des milieux de résidence, trois groupes de ménages se distinguent par leur taille et leur type et deux autres plutôt par les caractéristiques du chef de ménage (en milieu rural, sa scolarisation et son âge, en milieu urbain, le sexe et l'âge). Dans chacun des milieux, quatre ménages sur dix se retrouvent dans un profil qui peut être considéré comme le profil-type et qui nous servira de référence ; des profils peu fréquents, atypiques, apparaissent aussi dans chacun des milieux. Le ménage urbain type (Tableau A.I. en annexe) est nucléaire, de taille variable, mais qui ne dépasse que très rarement huit personnes ; le chef est toujours un homme, âgé entre 25 et 60 ans, dont le niveau de scolarisation est variable. Deux autres types se réfèrent à des ménages plutôt grands et deux plutôt petits; ces derniers se distinguent par les caractéristiques du chef de ménage. Un petit groupe de ménages atypiques (U5. 6\%) est de taille généralement très petite, souvent nucléaires, dans huit cas sur dix sans enfants et la moitié sans jeune ou adulte. Un autre groupe atypique, un peu plus fréquent $(U 4,12 \%)$ se distingue essentiellement par le fait qu'il est dirigé par une femme. C'est dans les deux autres groupes urbains, qui comptent chacun pour environ $20 \%$, que l'on trouve le plus de ménages élargis (à d'autres apparentés dans $U 2$, et à des tierces personnes dans U3). Ce dernier est souvent très grand, avec beaucoup d'enfants et de jeunes/adultes.

En milieu rural (Tableau A.2. en annexe), le profiltype $(\mathrm{RI})$ est de taille moyenne (3 à 8 personnes) ; il y a toujours au moins un jeune ou un adulte, mais dans un-tiers des cas aucun enfant; le chef de ménage est un homme qui n'a pas été à l'école. $\mathrm{Ce}$ profil-type s'oppose à deux petits groupes atypiques (R5. 9\% et R4. II\%) qui ont souvent un nombre très réduit de personnes, dans R5, souvent aucun adulte/jeune et dans R4, aucun enfant, et des chefs soit plus âgés (R5) soit plus jeunes (R4) que la norme. Les deux autres types se distinguent par leur taille et les caractéristiques du chef de ménage. Dans neuf cas sur dix, le type R2 (21\%) compte plus de huit personnes, dont de nombreux enfants et jeunes/adultes. Alors que R3 (I7\%) est le type de ménage rural qui compte le plus souvent un chef instruit.

Mesure de la qualité de vie

Dans les données du RGPH-2009, quatre types d'information permettent d'analyser les différences de qualité de vie : le confort du logement, la scolarisation des enfants, leur survie et la situation des femmes. Ce dernier point est l'objet d'une autre publication $^{\text {viii. }}$ A cause d'un important sous- 
enregistrement des naissances vivantes, nous renonçons ici à considérer la survie des enfants. La qualité de vie est ainsi prise en compte à travers la richesse mesurée par l'accès au confort moderne et un investissement durable à travers la scolarisation des enfants du ménage.

\section{Qualité du logement}

L'indice de qualité du logement a été construit en retenant pour le milieu urbain le type d'habitat, les principaux matériaux des murs, du toit et du sol, le principal mode d'éclairage, d'approvisionnement et de source d'énergie pour la cuisine, le type d'aisance, le statut d'occupation, l'existence du foyer amélioré, le mode d'évacuation des ordures et des eaux usées. Pour le milieu rural, certaines caractéristiques, jugées trop homogènes, ont été exclues (source d'énergie pour la cuisine, type d'aisance, statut d'occupation, mode d'évacuation des ordures et des eaux usées). Cet indice exprime donc l'accès au confort moderne (Garenne, 20I4). II a été construit séparément pour chacun des milieux à partir d'une analyse en composantes principales (ACP). Par la méthode des nuées dynamiques, dans chacun des milieux, trois groupes ont été identifiés à partir des résultats des deux premiers axes ${ }^{i x}$ : les ménages ayant la situation la moins bonne (case en banco, sol en terre battue, etc.), un groupe intermédiaire majoritaire et les mieux lotis (maisons en dur, toit en béton et sol cimenté,...).

\section{Scolarisation ajustée des enfants}

Les indicateurs de scolarisation sont élaborés pour chacun des trois niveaux d'enseignement, à savoir le Fondamental $\mathrm{I}$, le Fondamental 2 et le Secondaire. Les premier et second cycles de l'enseignement fondamental correspondent à l'école primaire et au secondaire inférieur, et concernent respectivement les groupes d'âges officiels 7-12 ans et I3-15 ans. Le cycle d'enseignement secondaire, c'est-à-dire le niveau secondaire supérieur, comprend les jeunes âgés de 16 à 18 ans. Ce troisième niveau n'est retenu que pour le milieu urbain, étant trop peu fréquent en milieu rural (4,7\% des enfants contre $27,7 \%$ en milieu urbain). Les calculs sont faits pour les ménages ayant au moins un enfant de l'âge scolarisable et séparément pour le milieu urbain et rural.

Pour chaque niveau de scolarisation, le nombre d'enfants scolarisés dans un ménage donné est comparé au nombre moyen d'enfants scolarisés parmi les ménages ayant le même nombre d'enfants scolarisables. Chaque ménage est donc situé par rapport à son groupe de référence, en rapportant le nombre d'enfants qu'il scolarise effectivement à la moyenne d'enfants scolarisés dans ce groupe ayant le même nombre d'enfants scolarisables. On obtient ainsi un indicateur de scolarisation ajustée qui permet d'évaluer l'effort relatif accompli par chaque ménage dans la scolarisation des enfants par rapport aux autres ménages ayant la même charge d'enfants. Avec $\mathrm{NS}_{\mathrm{ij}}$ le nombre d'enfants scolarisés dans le ménage $i$ ayant $j$ enfants scolarisables et $\mathrm{NMS}_{\text {tot, }}$ le nombre moyen d'enfants scolarisés dans la population totale des ménages ayant $\mathrm{j}$ enfants à scolariser, l'indicateur de la scolarisation ajustée pour le ménage i $\left(\mathrm{SA}_{\mathrm{i}}\right)$ se calcule comme suit:

$S A_{i}=\frac{N S_{i j}}{N M S_{t o t, j}} \quad$ Où $\mathrm{j}=1,2,3$ et 4,5 ou plus et $N M S_{t o t, j}=\frac{1}{n j} \sum_{k=1}^{n j} n_{j k}$

Pour chaque niveau, l'indicateur de scolarisation ajustée est regroupé en trois catégories : les ménages qui n'ont scolarisé aucun enfant (scolarisation nulle) et, parmi ceux qui en ont scolarisé au moins un, ceux qui se situent en-dessous (scolarisation moyenne) et au-dessus de la valeur médiane de l'indicateur (scolarisation élevée).

Nous ne comparons donc pas ici les niveaux de scolarisation, mais comment se situe le ménage par rapport aux autres ménages du même milieu ayant le même nombre d'enfants scolarisables.

Méthodes d'analyse : appréhender les inégalités entre les ménages

Nous examinons les avantages et désavantages des différents types de ménages, à savoir leur capacité à utiliser les ressources et surmonter les crises. L'objectif n'est pas d'analyser les niveaux atteints de bien-être, mais les inégalités entre les ménages et leur association avec les attributs du ménage. On considère donc que les ménages développent des stratégies distinctes selon leur composition et qu'elles se reflètent en des inégalités de qualité de vie. Prenant le ménage comme unité d'analyse, on examine dans quelle mesure les différences de qualité de vie - décrite ici par le confort moderne du logement et la scolarisation des enfants - sont attribuables aux attributs du ménage. On distingue deux types d'attributs (voir Tableau I): les opportunités offertes par la composition du ménage, synthétisée par les profils élaborés à travers l'analyse de cluster, et les caractéristiques modulant directement l'accès aux ressources, à savoir le nombre de personnes actives et celui de plus âgés et la présence de migrants. Par des analyses de régression logistique ${ }^{x}$, nous estimons les effets de l'appartenance aux différents types de ménages sur l'appartenance aux ménages qui ont un logement plus confortable et scolarisent mieux leurs enfants. Un second modèle contrôle pour l'effet des variables d'accès aux ressources. Dans une dernière partie, on 
examine dans quelle mesure certains ménages cumulent les avantages sur les deux dimensions.

Les analyses sont faites séparément pour le milieu urbain et le milieu rural pour atténuer le rôle des énormes écarts qui subsistent dans la disponibilité locale des ressources, en particulier de l'offre d'infrastructures et de services.

Résultats:

Types de ménages et qualité de vie

Types de ménages et qualité de logement

L'analyse de régression logistique sur les attributs qui accroissent l'appartenance aux ménages ayant un logement plus confortable (Tableau 3) met en évidence dans les deux milieux les avantages des plus grands ménages et, en milieu rural, encore plus, de ceux qui sont dirigés par un chef instruit. En milieu urbain, les ménages dirigés par une femme viennent en seconde position et c'est le ménage type qui apparaît désavantagé. En milieu rural, ce sont ceux dirigés par des chefs âgés qui sont les moins bien lotis.

En milieu urbain, le contrôle pour les attributs d'accès aux ressources ne modifie pas les inégalités selon les types de ménage. En revanche, en milieu rural, ceuxci accroissent l'avantage des très grands ménages et diminuent celui des ménages au chef instruit, comme aussi celui des très petits ménages. Ceci montre bien le rôle important, en milieu rural, des contributions individuelles des membres.

Dans les deux milieux, les attributs d'accès ont un effet propre, indépendamment du différentiel selon le type de ménage. La présence de migrants, particulièrement de migrants internes, accroît le risque statistique d'appartenir aux ménages les mieux logés, alors que le nombre d'enfants est négativement associé au confort - les ménages sans enfants étant les mieux lotis - comme aussi la présence d'âgés et, dans une moindre mesure, de seniors. Toutefois, dans le contexte rural, dominé par les activités agricoles, ce n'est pas le nombre de personnes déclarant une activité qui influe le confort : il aurait même un effet négatif. De même, la présence d'enfants travailleurs est associée négativement au confort du logement, mais l'effet est plus marqué en milieu rural qu'en milieu urbain.

Tableau 3 : Bonne qualité de logement selon le type de ménage par milieu de résidence (Rapport de cote)

\begin{tabular}{|c|c|c|c|c|c|c|}
\hline & \multicolumn{2}{|c|}{ Urbain } & \multicolumn{4}{|c|}{ Rural } \\
\hline Types de ménages & & & Types de ménages & & & \\
\hline Elargis autres parents & $\mathrm{I}, \mathrm{II}$ & $\mathrm{I}, 14$ & CM instruits & 3,61 & & 3,05 \\
\hline CM femme & 1,65 & 1,65 & Très grands & 1,86 & & 2,41 \\
\hline Urbain type (ref) & 1,00 & 1,00 & Rural type & 1,00 & & 1,00 \\
\hline Tpetits, nucléaires, $C M$ jeunes & 1,23 & 1,18 & CM âgés & 0,98 & ns & 0,96 \\
\hline Tgrands, élargis SL & 2,72 & 2,81 & $\begin{array}{l}\text { Tpetits, nucléaires, } \\
\text { CM jeunes }\end{array}$ & 2,18 & & $\mathrm{I}, 57$ \\
\hline \multicolumn{7}{|l|}{ Travail des enfants } \\
\hline Oui & \multirow{2}{*}{\multicolumn{2}{|c|}{$\begin{array}{l}0,85 \\
1,00\end{array}$}} & & & \multicolumn{2}{|c|}{0,44} \\
\hline $\begin{array}{l}\text { Non (ref) } \\
\text { Personnes occupées }\end{array}$ & & & & & \multicolumn{2}{|c|}{1,00} \\
\hline 0 & & 0,87 & & & \multicolumn{2}{|l|}{$\mathrm{I}, \mathrm{I}$} \\
\hline I à 2 (ref) & & 1,00 & & & \multicolumn{2}{|c|}{1,00} \\
\hline 3 ou plus & & 1,26 & & & \multicolumn{2}{|c|}{0,85} \\
\hline \multicolumn{7}{|l|}{ Emigrants } \\
\hline Oui & & 1,95 & & & \multicolumn{2}{|c|}{$\mathrm{I}, 15$} \\
\hline \multirow{2}{*}{\multicolumn{7}{|c|}{$\begin{array}{l}\text { Non (ref) } \\
\text { Migrants internes }\end{array}$}} \\
\hline & & & & & & \\
\hline Oui & & 2,59 & & & \multicolumn{2}{|c|}{4,42} \\
\hline & & 1,00 & & & 1,00 & \\
\hline \multicolumn{7}{|l|}{ Nombre d'enfants } \\
\hline 0 enf. & & 2,25 & & & \multicolumn{2}{|c|}{$|, 2|$} \\
\hline I enf. & & 1,66 & & & \multicolumn{2}{|c|}{1,16} \\
\hline 2 enf. & & 1,32 & & & \multicolumn{2}{|c|}{1,09} \\
\hline 3 enf.ou plus (ref) & & 1,00 & & & \multicolumn{2}{|c|}{1,00} \\
\hline \multicolumn{7}{|l|}{ Présence de personnes âgées } \\
\hline Oui & & 0,66 & & & \multicolumn{2}{|c|}{0,91} \\
\hline
\end{tabular}




\begin{tabular}{|lllll|}
\hline \multicolumn{2}{c}{ Urbain } & \multicolumn{2}{c|}{ Rural } \\
\hline $\begin{array}{llll}\text { Types de ménages } \\
\text { Non (ref) }\end{array}$ & & Types de ménages & \\
Présence de seniors & & 1,00 & & 1,00 \\
Oui & & 0,73 & & 0,97 \\
Non (ref) & & 1,00 & 0,09 & 0,07 \\
\hline Constante & 0,18 & 0,06 & 51723,9 & 169351,9 \\
Chi-deux du modèle & 12868,8 & 35733,5 & 0,05 & 0,17 \\
Nagelkerke R Square & 0,04 & 0,11 & & \\
\hline
\end{tabular}

Les rapports de cote sont significatifs au seuil de de $1 \%$ ou $5 \%$, sauf indication ns (non significatif).

Source : RGPH-2009, Calculs des auteurs

Types de ménage et scolarisation des enfants

Le risque statistique d'appartenir aux ménages dans lesquels la scolarisation des enfants est plus élevée est analysé par une analyse de régression logistique (Tableau 4) en examinant dans quelle mesure, dans chacun des milieux, les ménages se distinguent du ménage type. Le premier constat est que les écarts s'accroissent avec le niveau de scolarisation.

En milieu urbain, à tous les niveaux, les enfants des très petits ménages, dirigés par un chef jeune, sont nettement moins souvent parmi les mieux scolarisés que ceux des ménages urbains types et encore plus par rapport aux trois autres profils. C'est parmi les ménages élargis, très grands et grands, ainsi que ceux dirigés par une femme que la proportion de scolarisation élevée est plus marquée: respectivement, au niveau secondaire, cinq fois plus, près de quatre et trois fois plus que dans les ménages urbains types. Toutefois, les très grands ménages élargis perdent leur avantage lorsque l'on exclut de l'analyse les ménages qui ne scolarisent aucun enfant (calculs non présentés ici) : comme le montre aussi l'analyse bivariée, ils se retrouvent le plus souvent dans le groupe intermédiaire qui ne scolarise qu'une partie des enfants.

En milieu rural, c'est l'instruction du chef de ménage qui apparaît comme le facteur le plus important au niveau $\mathrm{FI}$, mais au niveau $\mathrm{F} 2$ ce sont les très grands ménages qui se distinguent le plus en faveur de la scolarisation. On observe aussi un fort désavantage des très petits ménages à chef jeune, et des ménages dirigés par des chefs âgés.

En milieu urbain, le contrôle pour l'effet des attributs $d$ 'accès aux ressources diminue légèrement la valeur des coefficients, mais sans changer fondamentalement les résultats. En milieu rural, l'introduction de ces variables accentue les avantages des très grands ménages et de ceux avec un CM instruit, et les désavantages des autres types.

L'effet net de ces variables d'accès aux ressources n'est pas très marqué mais s'accroît avec le niveau de scolarisation. Dans les deux milieux, la présence de femmes seniors est un facteur d'accroissement de la scolarisation, comme aussi celle de migrants internes. Au niveau F2 et secondaire, on observe aussi un léger effet négatif du nombre de personnes occupées.

Tableau 4. Scolarisation ajustée élevée selon le type de ménages par niveau d'enseignement et milieu de résidence (Rapports de cote)

\begin{tabular}{|c|c|c|c|c|c|c|c|c|c|c|c|}
\hline \multirow{2}{*}{ Variables } & \multicolumn{6}{|c|}{ Urbain } & \multirow{2}{*}{ Variables } & \multicolumn{4}{|l|}{ Rural } \\
\hline & \multicolumn{2}{|c|}{$\mathrm{FI}$} & \multicolumn{2}{|l|}{$\mathrm{F} 2$} & \multicolumn{2}{|l|}{$\mathrm{Sec}+$} & & \multicolumn{2}{|l|}{$\mathrm{FI}$} & \multicolumn{2}{|l|}{$\mathrm{F} 2$} \\
\hline \multicolumn{12}{|l|}{ Types de ménage } \\
\hline $\begin{array}{l}\text { Urbain type } \\
\text { (ref) }\end{array}$ & 1,00 & 1,00 & 1,00 & 1,00 & 1,00 & 1,00 & Rural type (ref) & 1,00 & 1,00 & 1,00 & 1,00 \\
\hline $\begin{array}{l}\text { Elargis autres } \\
\text { parents }\end{array}$ & ।,75 & 1,63 & 2,66 & 2,39 & 3,92 & 3,66 & CM instruits & 2,30 & 2,18 & 2,94 & 2,73 \\
\hline $\begin{array}{l}\text { CM femme } \\
\text { Tpetits, }\end{array}$ & $\mathrm{I}, 42$ & I,34 & 2,54 & 2,19 & 3,68 & 3,08 & Très grands & 1,37 & $|, 3|$ & 3,14 & 3,00 \\
\hline $\begin{array}{l}\text { nucléaires, CM } \\
\text { jeunes }\end{array}$ & 0,27 & 0,26 & 0,42 & 0,38 & 0,62 & 0,53 & CM âgés & 0,66 & 0,61 & 1,15 & 0,87 \\
\hline $\begin{array}{l}\text { Tgrands, élargis } \\
\text { SL }\end{array}$ & $\mathrm{I}, 33$ & $\mathrm{I}, 20$ & 3,92 & 3,57 & 5,18 & 5,02 & $\begin{array}{l}\text { Tpetits, } \\
\text { nucléaires CM } \\
\text { jeunes }\end{array}$ & 0,11 & 0,11 & 0,38 & 0,39 \\
\hline $\begin{array}{l}\text { Femmes Senior } \\
\text { Non (ref) }\end{array}$ & & 1,00 & & 1,00 & & $\mathrm{I}, 00$ & & & 1,00 & & 1,00 \\
\hline $\begin{array}{l}\text { Oui } \\
\text { Personnes âgées }\end{array}$ & & $|, 2|$ & & 1,59 & & $|, 7|$ & & & 1,21 & & 1,65 \\
\hline
\end{tabular}




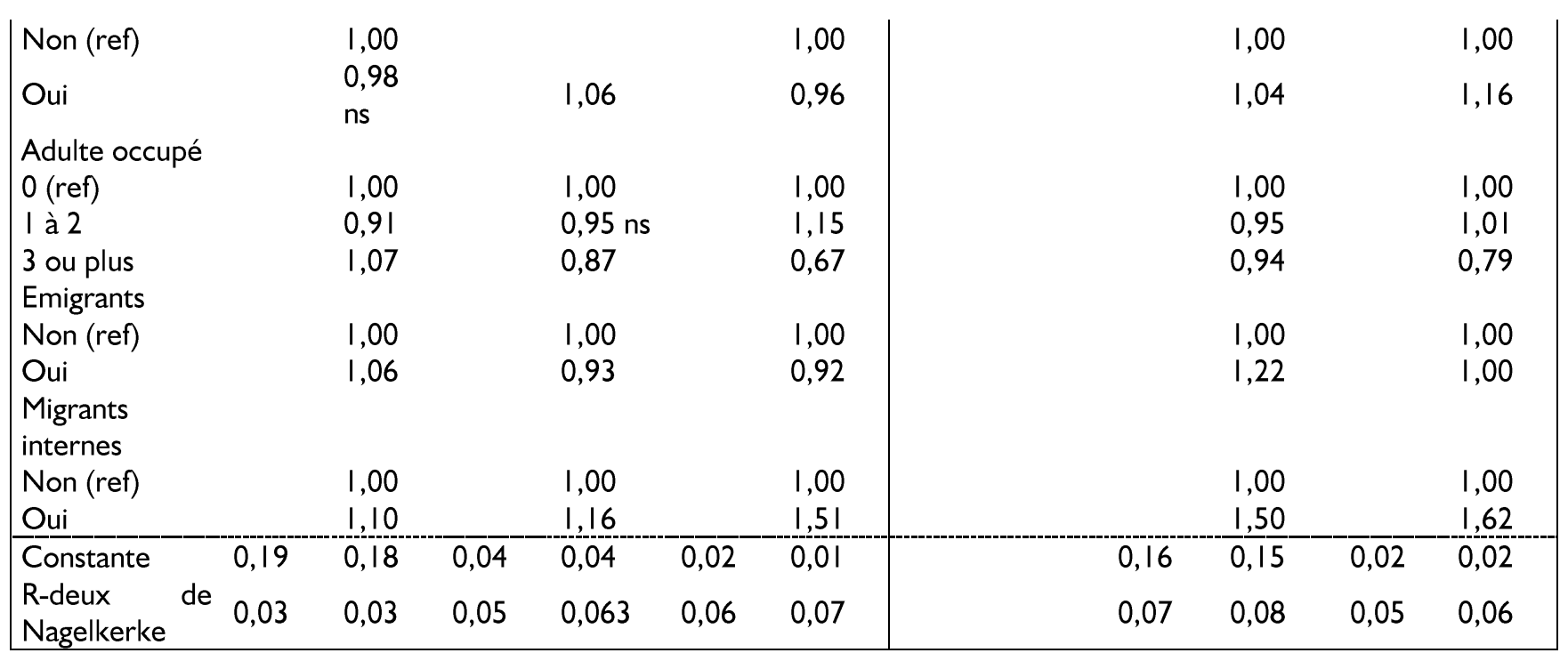

Les rapports de cote sont significatifs au seuil de de $1 \%$ ou $5 \%$, sauf indication ns (non significatif).

Source : RGPH-2009, Calculs des auteurs

\section{Cumul des avantages}

Finalement, on examine ici quels ménages sont parmi ceux qui, à la fois, ont un logement plus confortable et scolarisent le plus leurs enfants. Le premier constat est que ce n'est qu'une faible proportion qui cumule ces deux dimensions de la qualité de vie (pour la qualité du logement et la scolarisation au premier niveau fondamental respectivement $6 \%$ en milieu urbain et $4 \%$ en milieu rural et environ $2 \%$ et à peine $1 \%$ pour le cumul aux niveaux F2 et secondaire). Pour cette raison, nous retenons dans l'analyse qui suit les ménages qui ont au moins une des deux qualités examinées. C'est alors quatre ménages urbains sur dix et un quart des ménages ruraux qui sont parmi les privilégiés qui cumulent au fondamental $\mathrm{I}$, et respectivement un quart et $15 \%$ au F2, et un quart et $14 \%$ au secondaire. Ce sont alors les ménages urbains très grands élargis et les ménages ruraux dirigés par un chef instruit qui se démarquent considérablement des autres (Tableau $5)$.

Tableau 5. Cumul d'avantages en matière de qualité de logement et/ou de scolarisation selon le profil des ménages (fréquences)

\begin{tabular}{|c|c|c|c|c|c|c|c|c|c|c|c|c|}
\hline \multirow{2}{*}{$\begin{array}{l}\text { Bonne } \\
\text { qualité de } \\
\text { logement } \\
\text { (QL) ou } \\
\text { scolarisatio } \\
\text { n élevée }\end{array}$} & \multicolumn{6}{|c|}{ Profils ménages urbains (\% des ménages) } & \multicolumn{6}{|c|}{ Profils ménages ruraux (\% des ménages) } \\
\hline & $\begin{array}{l}\text { Élargis } \\
\text { autres } \\
\text { parent } \\
\mathrm{s}\end{array}$ & $\begin{array}{l}\text { CM } \\
\text { femme }\end{array}$ & $\begin{array}{l}\text { Urb. } \\
\text { type }\end{array}$ & $\begin{array}{l}\text { Tpt } \\
\text { nucl. } \\
\text { CM } \\
\text { jeune }\end{array}$ & $\begin{array}{l}\text { Tgd } \\
\text { élargi } \\
\text { SL }\end{array}$ & Ens. & $\begin{array}{l}\mathrm{CM} \\
\text { instruit }\end{array}$ & $\begin{array}{l}\text { Très } \\
\text { grand }\end{array}$ & $\begin{array}{l}\text { Rural } \\
\text { type }\end{array}$ & $\begin{array}{l}\text { CM } \\
\text { âgés }\end{array}$ & $\begin{array}{l}\text { Tpts, } \\
\text { nucl. } \\
\text { CM } \\
\text { jeune }\end{array}$ & Ens. \\
\hline $\begin{array}{ll}\mathrm{QL} & \text { ou } \\
\text { Scola FI }\end{array}$ & 48,0 & 47,4 & 36,3 & 23,7 & 59,2 & 44,3 & 42,1 & 27,8 & 20,0 & 16,6 & 17,7 & 24,8 \\
\hline $\begin{array}{l}\mathrm{QL} \\
\text { Scola F2 }\end{array}$ & 24,4 & 29,8 & 18,3 & 19,3 & 41,8 & 25,9 & 27,4 & 18,4 & 9,8 & 9,8 & 17,0 & 15,3 \\
\hline $\begin{array}{l}\text { QL ou } \\
\text { Scola sec }\end{array}$ & 24,8 & 29,7 & 16,7 & 18,9 & 43,2 & 25,7 & 25,3 & 15,9 & 8,6 & 8,3 & 16,6 & 13,7 \\
\hline
\end{tabular}

Source : RGPH-2009, Calculs des auteurs

L'analyse de régression, qui situe les ménages par rapport au ménage type de chacun des milieux, confirme l'avantage net, dans leur contexte, des très grands ménages élargis urbains et des ménages ruraux dirigés par un chef scolarisé. Toutefois, se distinguent également de façon très nette les ménages urbains dirigés par une femme et les très grands ménages ruraux (Tableau 6). Le contrôle pour les caractéristiques influençant l'accès aux ressources ne modifie pas fondamentalement les résultats. Toutefois, l'avantage net des deux types de ménages urbains élargis et des ménages ruraux dirigés par un chef instruit diminue quelque peu : il était donc en partie dû à la présence d'un plus grand nombre de 
membres contribuant au bien-être, à savoir les migrants et les seniors.

Tableau 6. Cumul des avantages en matière de qualité du logement ou de scolarisation des enfants selon le type de ménages par niveau de scolarisation et milieu de résidnece (Rapports de cotes)

\begin{tabular}{|c|c|c|c|c|c|c|}
\hline \multirow{2}{*}{$\begin{array}{l}\text { URBAIN } \\
\text { Types de ménage (Urbain type) }\end{array}$} & \multicolumn{2}{|c|}{ QL ou FI } & \multicolumn{2}{|c|}{ QL ou F2 } & \multicolumn{2}{|c|}{ QL ou Sec. } \\
\hline & & & & & & \\
\hline Elargis autres parents & 1,62 & $\mathrm{I}, 48$ & $\mathrm{I}, 44$ & 1,34 & 1,64 & $\mathrm{I}, 55$ \\
\hline CM femme & 1,58 & 1,58 & 1,90 & 1,92 & 2,11 & 2,14 \\
\hline Tpetits, nucléaires, CM jeunes & 0,55 & 0,52 & 1,07 & 1,11 & 1,16 & $1,2 \mid$ \\
\hline T grands, élargis SL & 2,56 & 2,23 & 3,20 & 2,80 & 3,80 & 3,33 \\
\hline \multicolumn{7}{|l|}{ Accès aux ressources } \\
\hline Personnes âgées Oui (Non) & & 0,83 & & 0,80 & & 0,79 \\
\hline Présence de seniors Oui (Non) & & 1,32 & & 1,08 & & 1,05 \\
\hline Emigrants Oui (Non) & & $\mathrm{I}, 44$ & & 1,59 & & 1,59 \\
\hline Migrants internes Oui (Non) & & 1,56 & & 2,11 & & 2,36 \\
\hline Enfants travailleur Oui (Non) & & 0,57 & & 0,67 & & 0,74 \\
\hline \multicolumn{7}{|l|}{ Occupation (2 à 3) } \\
\hline 0 & & 0,86 & & 0,91 & & 0,95 \\
\hline 1 à 2 & & 1,00 & & 1,00 & & 1,00 \\
\hline 3 ou plus & & 1,09 & & 1,12 & & 1,10 \\
\hline Constante & 0,57 & 0,43 & 0,23 & 0,14 & 0,20 & 0,12 \\
\hline R-deux de Nagelkerke & 0,053 & 0,083 & 0,054 & 0,092 & 0,068 & 0,111 \\
\hline \multicolumn{7}{|l|}{ RURAL } \\
\hline \multicolumn{7}{|l|}{ Types de ménage (Rural type) } \\
\hline CM instruit & 2,91 & 2,66 & 3,49 & 3,02 & & \\
\hline Très grands & $\mathrm{I}, 54$ & 1,86 & 2,08 & 2,48 & & \\
\hline CM âgés & 0,79 & 0,71 & $\mathrm{I}, 0 \mathrm{l} \mathrm{ns}$ & 0,95 & & \\
\hline T. petits & 0,87 & 0,70 & 1,89 & 1,53 & & \\
\hline \multicolumn{7}{|l|}{ Accès aux ressources } \\
\hline Personnes âgées Oui (Non) & & 0,99 & & 0,99 & & \\
\hline Présence de seniors Oui (Non) & & 1,24 & & 1,16 & & \\
\hline Émigrants Oui (Non) & & 1,24 & & 1,14 & & \\
\hline Migrants internes Oui (Non) & & 2,61 & & 3,78 & & \\
\hline Enfants travailleurs Oui (Non) & & 0,33 & & 0,44 & & \\
\hline \multicolumn{7}{|l|}{ Occupation (2 à 3) } \\
\hline 0 & & $0,99 \mathrm{~ns}$ & & 1,09 & & \\
\hline 1 à 2 & & 1,00 & & 1,00 & & \\
\hline 3 ou plus & & $\mathrm{I}, 00 \mathrm{~ns}$ & & 0,86 & & \\
\hline Constante & 0,25 & 0,24 & 0,11 & 0,09 & & \\
\hline R-deux de Nagelkerke & 0,053 & 0,147 & 0,053 & 0,155 & & \\
\hline
\end{tabular}

Les rapports de cote sont significatifs au seuil de de $1 \%$ ou $5 \%$, sauf indication ns (non significatif).

Source : RGPH-2009, Calculs des auteurs

\section{Discussion}

En analysant la relation entre la composition du ménage et la qualité de vie, ce travail s'interroge sur la question de savoir si une descendance nombreuse peut être interprétée comme une stratégie de subsistance qui expliquerait donc le maintien d'une fécondité élevée au Mali. Les résultats montrent certes que les grands ménages sont globalement parmi les mieux lotis en matière de qualité de leur logement et de scolarisation des enfants. Toutefois, tant en milieu urbain que rural, un plus grand nombre d'enfants est au contraire associé à un confort moindre. Ceci va dans le sens de la théorie du coût des enfants. Les enfants ne constituent plus une source de richesse et leur travail, négativement associé au bien-être serait plutôt l'expression des besoins des plus démunis.

Plus que la taille du ménage, c'est leur capacité à utiliser les ressources du contexte pour vivre une vie de qualité que l'on examine ici. Le ménage est donc considéré comme un facteur collectif de conversion, pour reprendre la terminologie de l'approche par les Capabilités. On examine ainsi deux thèses 
antagonistes largement répandues dans la littérature. D'une part, le postulat selon lequel les grands ménages peuvent diversifier leurs stratégies de subsistance et donc devraient avoir de meilleures conditions de vie. D'autre part, celui selon lequel les ménages nucléaires, plus modernes, auraient un meilleur accès aux institutions, et, plus homogènes, seraient plus égalitaires.

Nous avons donc développé une typologie des ménages qui prend en compte non seulement leur taille, mais aussi leur composition. On a identifié dans chacun des deux milieux, un ménage-type qui se distingue de quatre autres profils moins fréquents. En milieu urbain, le ménage-type est de petite taille, généralement nucléaire et, en milieu rural, de taille intermédiaire, nucléaire un peu plus souvent que la moyenne. Dans les deux cas, le chef de ménage est un homme et, en milieu rural, il n'est pas instruit. Dans les deux contextes, ces ménages-type se distinguent par une qualité de vie moindre que dans les autres, à l'exception des types les plus marginaux, très petits ménages dirigés par des chefs plus jeunes (ou, en milieu rural, plus âgés).

\section{Avantage des grands ménages}

Dans les deux contextes, globalement les grands ménages élargis apparaissent avoir une meilleure qualité de vie, tel qu'exprimée par le confort du logement et la scolarisation des enfants. L'avantage des grands ménages serait associé à une opportunité de diversification des stratégies de subsistance. C'est ainsi que le contrôle pour la présence de personnes susceptibles de contribuer au bien-être du ménage diminue un peu leur avantage. On observe en effet que, quel que soit le type de ménage, la présence de migrants a un impact positif important, particulièrement en matière de confort du logement, et en milieu rural essentiellement les migrants internes. En matière de scolarisation, on constate aussi le rôle positif des personnes externes au noyau familial - apparentées ou non au chef de ménage - et un effet net de la présence de femmes senior. Ce dernier point va dans le sens de ce que Marcoux (1994) avait déjà observé, à savoir une meilleure scolarisation des enfants lorsqu'il y a suffisamment de femmes pour assumer les tâches domestiques.

Néanmoins, il convient de nuancer cette image de l'avantage des grands ménages élargis. Certes dans les deux milieux, ils apparaissent avoir une qualité de vie meilleure que les ménages-types. Toutefois, en milieu urbain, ce sont plus spécifiquement les grands ménages élargis accueillant aussi des personnes tierces sans lien de parenté avec le chef de ménage qui sont avantagés, plus que les ménages élargis n'accueillant que des membres de la parenté. En matière de qualité de vie, telle qu'exprimée par le cumul des deux dimensions retenues ici, ces derniers, comme aussi les ménages élargis ruraux qui ne comprennent guère de personnes non apparentées, sont dépassés par les ménages respectivement dirigés par une femme et par un chef instruit. Rappelons cependant que cet avantage des ménages urbains élargis à des tiers non apparentés n'est pas à interpréter comme le simple résultat de la contribution de ceux-ci, puisque ce sont souvent les ménages déjà plus riches qui peuvent accueillir des tiers ou engager des employés. Finalement, notons aussi que les apparentés cohabitant sont souvent des aînés et que la présence de personnes senior ou âgées diminuerait légèrement le confort, effet moindre en milieu rural où donc ils seraient moins à charge.

Le rôle des caractéristiques du chef de ménage

La typologie met en évidence les ménages dont le chef est atypique : en milieu urbain, eu égard à son sexe et, en milieu rural, à son niveau d'instruction.

En milieu urbain, les ménages dirigés par une femme se distinguent par un meilleur confort, mais surtout un accès accru des enfants à la scolarisation secondaire. En matière de cumul des deux dimensions de qualité de vie, ils dépassent les ménages élargis aux apparentés. Rappelons, toutefois, que les analyses bivariées avaient montré une distribution en $U$, à savoir que ces ménages étaient aussi un peu plus fréquemment que la moyenne parmi les moins favorisés selon ces deux dimensions. Ainsi, on aurait donc plusieurs types de ménages dirigés par une femme. Dans le contexte du Mali, il peut s'agir de femmes qui sont chefs de ménage du fait de leur autonomie, de la migration masculine ou de la polygamie sans coprésidence et non pas seulement des ménages dirigés par les femmes veuves ou séparées de leur mari. Les ménages dirigés par les femmes ne sont donc pas systématiquement vulnérables contrairement à ce qui est souvent mentionné, mais qui rejoint d'autres travaux par exemple Chant (2007) en gmabie , Philippine et Costa Rica ou Oginni et al (20/3) au Nigeria.

En milieu rural, dans un contexte où la scolarisation est peu répandue dans la population adulte, les ménages dirigés par un chef instruit ont un avantage significatif, tant en matière de confort du logement que d'instruction des enfants. Ce sont ceux qui cumulent le plus souvent ces deux dimensions de la qualité de vie, dépassant largement les très grands ménages. Ils sont trois fois plus nombreux que ceuxci à cumuler un logement confortable et une scolarisation élevée au premier niveau fondamental et quatre fois plus en prenant en compte la scolarisation au second niveau. 
Le mythe du petit ménage nucléaire progressiste n'est pas vérifié

Finalement, les résultats montrent que dans les deux milieux, les très petits ménages, dirigés généralement par un jeune chef ne se distinguent guère du ménage type en matière de confort et sont même nettement défavorisés en matière de scolarisation, particulièrement au niveau primaire. Dans les deux milieux, les très petits ménages nucléaires sont ceux qui se retrouvent le moins souvent parmi les niveaux les plus élevés dans les deux dimensions de qualité de vie. On peut aussi relever que les ménages ruraux qui jouissent d'une meilleure qualité de vie, à savoir ceux dirigés par un chef instruit, sont (avec les très grands ménages) parmi ceux qui sont le moins souvent nucléaires.

La thèse du ménage nucléaire progressiste n'est pas vérifiée. Les ménages nucléaires ne sont pas rares, représentant la moitié des ménages urbains et les deux-tiers des ménages ruraux. On les retrouve majoritairement dans les ménages-types qui, comme nous l'avons vu ne sont pas parmi les mieux lotis, mais aussi dans le groupe défavorisé des très petits ménages aux caractéristiques marginales.

\section{Conclusion}

Grâce à leur exhaustivité, les données du recensement nous ont permis de réaliser une analyse fine de la composition des ménages, en distinguant le milieu urbain du milieu rural. En revanche, ces données ne fournissent pas des informations détaillées sur la qualité de vie que nous mesurons ici à travers le confort du logement et la scolarisation des enfants. A cet égard, les grands ménages élargis apparaissent avoir un certain avantage. Le maintien d'une fécondité élevée fait probablement partie des stratégies familiales. Toutefois, les enfants semblent représenter plutôt un investissement à moyen terme qu'un appoint immédiat. Ce n'est pas la fécondité qui compte, mais son association aux modes de vie qui favorisent les familles élargies et l'accueil de tierces personnes. Finalement, la thèse de plus d'inégalités dans les plus grands ménages est vérifiée dans les deux milieux : les très grands ménages urbains élargis à des personnes non apparentées et les très grands ménages ruraux se retrouvent moins souvent parmi ceux qui ne scolarisent aucun enfant, mais rarement parmi ceux qui les scolarisent quasiment tous.

Les résultats confirment l'importance des caractéristiques du chef de ménage. Ainsi, dans le contexte rural de faible accès à l'école, le niveau d'instruction du chef de ménage se révèle être le facteur principal d'amélioration de la qualité de vie des ménages. Par ailleurs, contrairement à ce qu'on pourrait penser, les ménages urbains dirigés par une femme ne sont pas systématiquement plus vulnérables et se situent même juste après les grands ménages élargis en termes de qualité de vie.

II est toutefois évident que les données de recensement ne permettent pas une analyse approfondie des stratégies que développent les divers types de ménage. Seule une enquête spécifique approfondie recourant à des méthodes mixtes permettrait de mieux comprendre les avantages des plus grands ménages, la diversité des situations des ménages dirigés par une femme et la vulnérabilité des très petits ménages.

\section{Références}

Ariès, P (1960), L'enfant et la vie familiale sous I'Ancien régime. Paris: Plon.

Batterbury, S 2007, "Monde rural et transformations agraires au Sud : débat et défis", Actes du Colloque International PRIPODE, Paris, CICRED : 177-I84.

Billari, F and Wilson C 200I, "Convergence towards diversity? Cohort dynamics in the transition to adulthood in contemporary Western Europe", Max Plank Institue for Demographic Research. Rostock, Germany. Working Paper 200I-039. Available http://www.demogr.mpg.de/

Bongaarts, J 2006, "The causes of stalling fertility transitions". Studies in Family Planning 37, (I): III 6.

Caldwell, J.C (1982), The theory of fertility decline. London : Academic Press.

Calvès E.A et Marcoux R 2007, "Les processus d'individualisation «à l'africaine». Sociologie et sociétés $39,(2): 5-18$.

Chant, S. 2007, "Children in female-headed households". Lonodn School of Economics, Gender Institute New Working Paper Series Nol9, 107 p.

Cosio-Zavala, M.E 200I, "Les deux modèles de transition démographique en Amérique latine : le malthusianisme de la pauvreté", Transitions Démographiques des Pays du Sud, Gendreau F. et M. Poupard (éd.), Paris: Editions Estem : 4I-52

Garenne, M 2009, "Situations of fertility stall in Sub_Saharan Africa", African Population Studies 23, (2): I75-188.

Garenne, M 2013, "Stagnation dans les transitions de la fécondité : études de cas en Afrique subsaharienne" in: Tabutin D et Masquelier B (eds) : Ralentissements, résistances et ruptures dans les transitions démographiques, Chaire Quetelet 2010, Presses universitaires de UCL : 3I-46.

INSTAT (à paraître), Configuration des ménages et qualité de vie. Les avantages et désavantages des 
grands ménages au Mali. Institut National de la Statistique, Bamako (Mali).

Lee, G.L (2nd ed, 1999), Comparative perspectives, in Sussman et al (eds) Handbook of Marriage and Family, New York: Plenum Press.

LeGrand, T, Koppenhaver T, Mondain N, Gomis D, Randall S and Hill K 2003, "Reassessing the insurance effect : A qualitative analysis of fertility

Locoh, T et Mouvagha-Sow M 2005, "Vers de nouveaux modèles familiaux en Afrique de l'Ouest ?", Communication au XXVè Congrès international de la population, Tours.

Marcoux, R 1994, "Le travail ou l'école. L'activité des enfants et les caractéristiques des ménages en milieu urbain au Mali", Mali, Éditions du CERPOD.

Marie, A (éd., 1997), L'Afrique des individus, Itinéraires citadins dans I'Afrique contemporaine, Paris: Karthala.

Nussbaum M and Sen A.K (ed, 1993): The quality of life, Wider studies in development economics, Oxford: Clarendon Press.

Oginni, A, B. Ahonsi and F.Ukwuije (20l3), Are female-headed households typically poorer than male-heade households in Nigeria? Journal of Socio-economics, 45, I32-I 37.

Pilon, M et Vignikin K 1996, "Stratégies face à la crise et changements dans les structures familiales", Chapitrel 8 in: Coussy et Vallin J (eds), Crise et population en Afrique, Études du CEPED No. 13.

Robeyns, I 2005, "The capability approach : a theoretical survey", Journal of human development 6, (I) : 93-II4.

Sandron, F 20I3, "Les facteurs socio-économiques de la stagnation de la baisse de la fécondité : une revue de la littérature", in: Tabutin $D$ et Masquelier B (eds) : Ralentissements, résistances et ruptures dans les transitions démographiques, Chaire Quetelet 2010, Presses universitaires de UCL : I3-30.

Sauvain-Dugerdil C, Kalmykova N, Guang G.H, Ritschard Gi, Olszak M et Hagmann H-M 1997, behaviour in Senegal and Zimbabwe", Population and Development Review 29, (3): 375-403.

Leridon, $\mathrm{H}$ (ed, 20l4), Les théories de la fécondité. Textes fondamentaux. Série Manuels. INED, Paris. Locoh, T 2002, "Structures familiales et évolutions de la fécondité dans les pays à fécondité intermédiaire d'Afrique de l'Ouest", Expert group meeting on completing the fertility transition, Population Division, New York. UN/POP/CFT/2002/BP/7

"Vivre sa vieillesse en Suisse. Les transformations des modes de résidence des personnes âgées", European Journal of Population 13, (2): I-43.

Sauvain-Dugerdil C 2014, "Cibler les inégalités: Apport de l'approche des Capabilités aux études de population", chapitre 2 in: Sauvain-Dugerdil C (ed) : Application de l'approche des Capabilités de Sen à l'analyse démographique en Afrique, Etudes de la population africaine 28, (2): 72I-73I. http://aps.journals.ac.za/pub/issue/archive

Shapiro D, Kreider A, Varner C and Sinha M 2013, "Stalling of fertility transitions and socioeconomic changes in the developing world: evidence from the Demographic and Hesith Surveys", in: Tabutin $D$ et Masquelier B (eds) : Ralentissements, résistances et ruptures dans les transitions démographiques, Chaire Quetelet 2010, Presses univ. de UCL : 47-64.

Schoumaker B et Tabutin D (1999), Relation entre pauvreté et fécondité dans les pays du Sud. Connaissances, méthodologie et illustrations. Document de Travail No. 2, Février 1999, Université catholique de Louvain, Département des Sciences de la Population et du Développement

Sen, A.K (1999), Development as freedom, New York: Alfred A. Knopf Press

Toulmin, C (1992), Cattle, women and wells. Oxford Van de Kaa, D 1996, "Anchored Narratives: The Story and Findings of Half a Century of Research into the Determinants of Fertility", Population Studies 50, (3) : 389-432.

\section{ANNEXES}

Tableau A. I. Profils des ménages urbains (analyse de clusters)

\begin{tabular}{|c|c|c|c|c|c|c|c|}
\hline \multirow[b]{2}{*}{ Variables } & \multirow[b]{2}{*}{ Modalités } & \multicolumn{5}{|c|}{ Clusters Urbains } & \multirow[b]{2}{*}{ Total } \\
\hline & & $\begin{array}{l}\text { Ul. } \\
\text { Urbain- } \\
\text { type }\end{array}$ & $\begin{array}{c}\text { U2. } \\
\text { Élargis à } \\
\text { autres } \\
\text { parents }\end{array}$ & $\begin{array}{c}\text { U3. } \\
\text { Tgrands, } \\
\text { élargis à SL }\end{array}$ & $\begin{array}{c}\text { U4. } \\
\text { CM } \\
\text { femmes }\end{array}$ & $\begin{array}{c}\text { U5. } \\
\text { Tpetits, } \\
\text { nucl., } \\
\text { CM jeunes } \\
\text { ou aînés }\end{array}$ & \\
\hline $\begin{array}{l}\text { Taille du } \\
\text { ménage }\end{array}$ & $\begin{array}{l}\text { Très petit ( } \mathrm{I} \text { à } \\
2 \text { ) }\end{array}$ & 21,3 & 3,2 & 0,4 & 16,8 & 81,6 & 15,7 \\
\hline
\end{tabular}




\begin{tabular}{|c|c|c|c|c|c|c|c|}
\hline & $\begin{array}{l}\text { Petit (3 à 5) } \\
\text { Grand (6 à } 8 \text { ) }\end{array}$ & $\begin{array}{l}54,8 \\
23,6\end{array}$ & $\begin{array}{l}28,4 \\
42,4\end{array}$ & $\begin{array}{c}9,5 \\
18,8\end{array}$ & $\begin{array}{l}42,1 \\
24,9\end{array}$ & $\begin{array}{c}13,7 \\
4,2\end{array}$ & $\begin{array}{l}35,1 \\
26,5\end{array}$ \\
\hline & $\begin{array}{l}\text { Très grand } \\
(9+)\end{array}$ & 0,3 & 26,1 & 71,3 & 16,1 & 0,5 & 22,7 \\
\hline & Nucléaire & 97,3 & 4,6 & 20,3 & 37,3 & 81,4 & 50,2 \\
\hline Type de & $\begin{array}{l}\text { Élargi à autres } \\
\text { parents }\end{array}$ & 0,1 & 94,0 & 26,1 & 45,4 & 15,8 & 35,8 \\
\hline & $\begin{array}{l}\text { El. AP et/ou } \\
\text { sans lien }\end{array}$ & 2,6 & I,4 & 53,6 & 17,3 & 2,8 & 14,0 \\
\hline Nombre & 0 & 53,7 & 29,3 & 14,2 & 37,4 & 83,8 & 39,6 \\
\hline d'enfants & 1 à 2 & 37,0 & 54,7 & 26,5 & 45,1 & 13,0 & 39,0 \\
\hline (6-I4 ans) & 3 à plus & 9,4 & 16,0 & 59,3 & 17,4 & 3,1 & 21,4 \\
\hline Nb jeunes/ & 0 & 0,0 & 0,1 & 0,0 & 2,4 & 54,1 & 3,6 \\
\hline adultes & 1 à 2 & 91,9 & 32,7 & 10,1 & 55,2 & 44,7 & 53,4 \\
\hline (I5-44 ans) & 3 à plus & 8,1 & 67,3 & 89,9 & 42,4 & $\mathrm{I}, \mathrm{I}$ & 43,0 \\
\hline Sexe du & Homme & 100,0 & 100,0 & 100,0 & 0,0 & 85,6 & 87,0 \\
\hline CM & Femme & 0,0 & 0,0 & 0,0 & 100,0 & 14,4 & 13,0 \\
\hline Niveau & $\begin{array}{l}\text { Aucun } \\
\text { Primaire }\end{array}$ & $\begin{array}{l}54,4 \\
16,9\end{array}$ & $\begin{array}{l}47,4 \\
22,5\end{array}$ & $\begin{array}{c}39,7 \\
6,6\end{array}$ & $\begin{array}{l}61,7 \\
13,7\end{array}$ & $\begin{array}{l}58,2 \\
11,2\end{array}$ & $\begin{array}{l}50,8 \\
15,6\end{array}$ \\
\hline$n$ du $C M$ & $\begin{array}{ll}\text { Sec. } & \text { ou } \\
\text { supérieur } & \end{array}$ & 28,8 & 30,1 & 53,7 & 24,6 & 30,6 & 33,6 \\
\hline & $\begin{array}{l}\text { Moins de } 25 \\
\text { ans }\end{array}$ & $\mathrm{I}, \mathrm{I}$ & 2,6 & 0,7 & 9,0 & 34,3 & 4,4 \\
\hline Age du CM & $25-44$ ans & 76,2 & 51,5 & 36,8 & 42,2 & 0,0 & 53,4 \\
\hline & $45-59$ ans & 17,8 & 24,9 & 48,7 & 29,9 & 35,5 & 28,2 \\
\hline & 60 ans ou plus & 4,9 & 21,0 & 13,8 & 18,9 & 30,2 & 14,0 \\
\hline $\begin{array}{l}\text { Total } \\
\text { Effectif } \\
\text { Pourcent }\end{array}$ & & $\begin{array}{c}100,0 \\
184974 \\
36,5\end{array}$ & $\begin{array}{c}\mid 00,0 \\
|3087| \\
25,8\end{array}$ & $\begin{array}{c}100,0 \\
98290 \\
19,4\end{array}$ & $\begin{array}{c}100,0 \\
61463 \\
12,1\end{array}$ & $\begin{array}{c}100,0 \\
30961 \\
6,1\end{array}$ & $\begin{array}{c}100,0 \\
506559 \\
100,0\end{array}$ \\
\hline & Valeurs les plus & levées & & Valeur & es plus fa & & \\
\hline
\end{tabular}

Tableau A.2. Profils des ménages ruraux (analyse de clusters)

\begin{tabular}{|c|c|c|c|c|c|c|c|}
\hline \multirow[b]{2}{*}{ Variables } & \multirow[b]{2}{*}{ Modalités } & \multicolumn{5}{|c|}{ Clusters ruraux } & \multirow[b]{2}{*}{ Total } \\
\hline & & $\begin{array}{l}\text { RI. } \\
\text { Rural- } \\
\text { type }\end{array}$ & $\begin{array}{c}\text { R2. } \\
\text { Très } \\
\text { grands } \\
\end{array}$ & $\begin{array}{c}\mathrm{R} 3 . \\
\mathrm{CM} \\
\text { instruit }\end{array}$ & $\begin{array}{l}\text { R4.Tpetits, } \\
\text { nucléaire, } \\
\text { CM jeune }\end{array}$ & $\begin{array}{l}\text { R5. } \\
\text { CM } \\
\text { âgés }\end{array}$ & \\
\hline \multirow[b]{2}{*}{$\begin{array}{l}\text { Taille } \\
\text { ménage }\end{array}$} & Très petit (I à 2) & 0,3 & 0,0 & 0,9 & 87,3 & 46,7 & 13,5 \\
\hline & $\begin{array}{l}\text { Petit ( } 3 \text { à } 5) \\
\text { Grand }(6 \text { à } 8) \\
\text { Très grand }(9+)\end{array}$ & $\begin{array}{c}56,2 \\
42,5 \\
0,9\end{array}$ & $\begin{array}{l}1,5 \\
7,2 \\
91,3\end{array}$ & $\begin{array}{c}58,2 \\
31,4 \\
9,6\end{array}$ & $\begin{array}{l}4,0 \\
8,7 \\
0,1\end{array}$ & $\begin{array}{c}34,1 \\
18,2 \\
1,0\end{array}$ & $\begin{array}{l}37,5 \\
27,6 \\
21,4\end{array}$ \\
\hline \multirow{3}{*}{ Type de ménage } & Nucléaire & 72,1 & 43,6 & 59,3 & 87,6 & 74,6 & 65,8 \\
\hline & $\begin{array}{l}\text { Élargi à autres } \\
\text { parents }\end{array}$ & 27,0 & 49,7 & 34,9 & 11,8 & 23,9 & 31,2 \\
\hline & $\begin{array}{l}\text { El. AP et/ou sans } \\
\text { lien }\end{array}$ & 0,9 & 6,7 & 5,8 & 0,6 & 1,5 & 3,0 \\
\hline
\end{tabular}




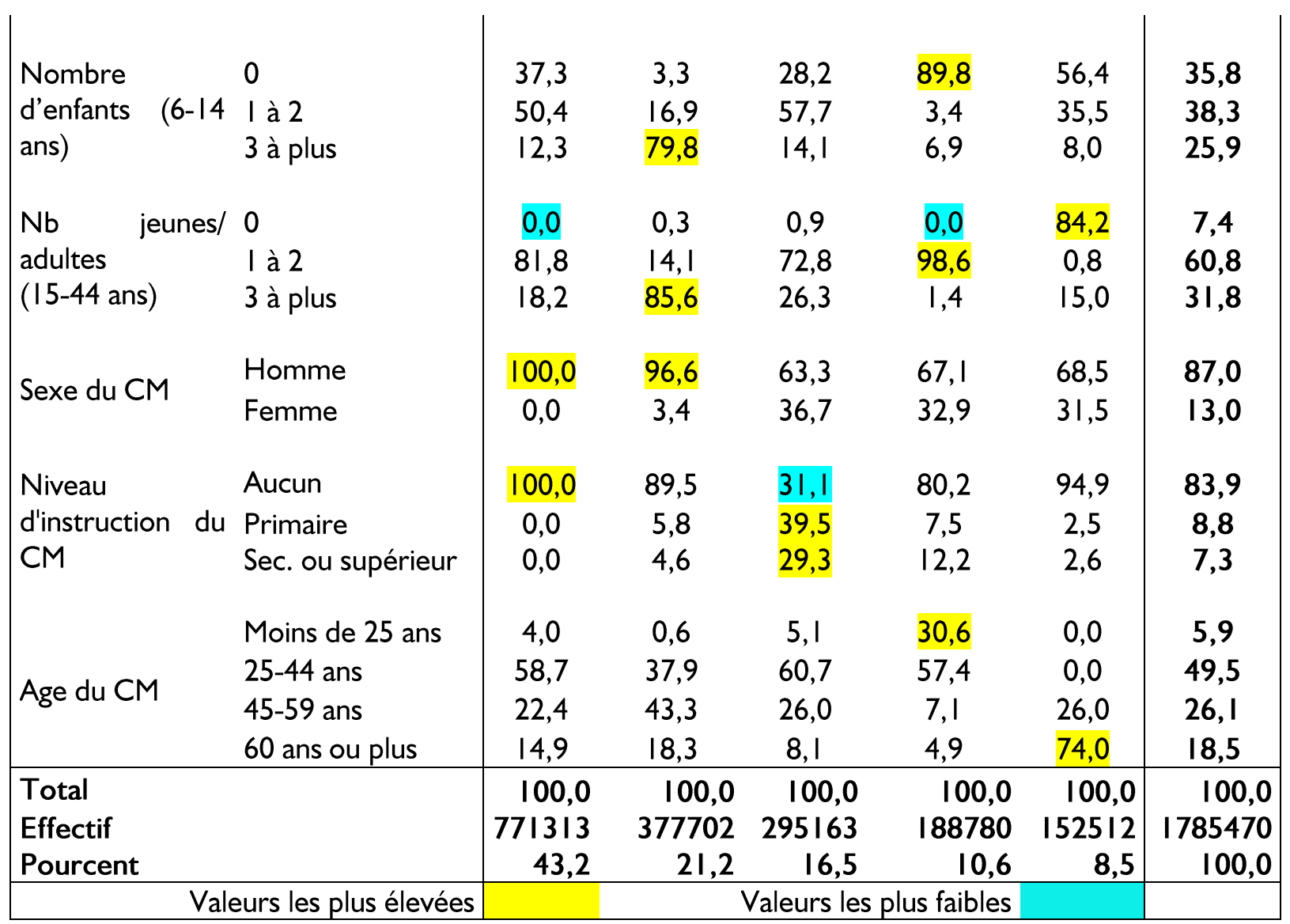


i. En milieu urbain, EDSM I 1981-83: 6,85, EDSM II 1984-86: 6,09, EDSM III 1995-96: 5,4, EDSM IV 2001 : 5,5. L'EDSM V 2012-13 donne un chiffre de 5, mais rappelons que cette enquête n'a pas couvert les régions du nord du pays. Les données du Recensement de 2009 établissent, avec des méthodes indirectes, un niveau total de 6,4 , et de 5 en milieu urbain, données entachées par un problème de sous-enregistrement des naissances.

ii. INSTAT (2012): Ménages et habitation. Rapport thématique 10. Bamako.

iii. Thème 1. Évaluation de la qualité des données. INSTAT, Bamako, décembre 2013

iv. Valeurs du quartile 1 et 3 (Tableau 1)

v. Réponses à la question: «Durant les 4 dernières semaines que faisait (nom) la plupart du temps ?» Les enfants travailleurs sont tous les enfants répondant aux modalités 1 (occupés), 2 (chômeur) et 3 (inactivité saisonnière). Pour les personnes de 15 ans et plus, il s'agit des modalités 1 et 3 (Tableau 1).

vi. L’émigration est appréhendée par la question : « $\mathrm{Au}$ cours des cinq dernières années, y a-t-il eu des membres de votre ménage qui se sont installés à l'étranger ?» Elle se réfère donc aux individus qui étaient hors du Mali au moment du recensement d'avril 2009. La migration interne concerne toutes les personnes recensées ayant résidé au moins six mois dans une circonscription administrative (commune, cercle, région) différente de celle de leur résidence habituelle ou qui ont résidé au moins 6 mois hors du Mali (pour la migration internationale).

vii. Voir le détail de l'analyse dans le chapitre 2 Attributs des ménages, Rapport INSTAT, à paraître.

viii. Sauvain-Dugerdil C., Abdoul M. Nouhou, Siaka Cissé et Assa G. Doumdia, (2016). "Configurations familiales et situation des femmes : le cas du Mali à travers les données du recensement", XIXe colloque international de l'Association internationale des démographes de langue française (AIDELF), Strasbourg.

ix. Cette méthode de partitionnement par agrégation à partir de noyaux permet de traiter de grands ensembles de données (plusieurs milliers d'individus); le nombre de classes souhaitées est déterminé à priori.

${ }^{\mathrm{x}}$. Les associations au niveau descriptif, analyses bivariées, ne sont pas reproduites ici. Elles figurent dans le rapport INSTAT, Chapitres 3 et 4. 